\title{
Definição de indicadores para a implantação de consórcios públicos intermunicipais para gestão de resíduos sólidos
}

O estudo teve como objetivo definir indicadores que possibilitarão a análise da viabilidade da implantação de consórcios públicos intermunicipais para a gestão dos resíduos sólidos. Para a definição dos mesmos foi utilizado o Método Delphi com a participação de 67 especialistas de todas as regiões do país. Após a realização prévia de uma seleção de indicadores a partir de estudos bibliográficos, os especialistas foram consultados em duas etapas, sendo aplicado instrumentos da estatística descritiva. Ao término da consulta foram definidos 30 indicadores, divididos em 5 dimensões, sendo: 9 na dimensão técnica, 3 na dimensão econômica, 9 na dimensão ambiental, 5 na dimensão social e 4 na dimensão política. Posteriormente, os indicadores foram aplicados em dois municípios do estado do Amazonas, através encaminhamento de formulário com os gestores municipais. Após obtenção dos dados e aplicados os respectivos pesos, obteve-se um percentual de $82,4 \%$ de necessidade/oportunidade para implantação de um consórcio entre os dois municípios. Contudo, há necessidade de ratificar esse resultado com dados primários e levantamento dos custos relativos.

Palavras-chave: Indicador; Resíduo; Sustentabilidade; Amazônia.

\section{Definition of indicators for the implementation of intercity public consortia for solid waste management}

\begin{abstract}
The study aimed to define indicators that will make it possible to analyze the feasibility of implementing public inter-municipal consortia for the management of solid waste. To define them, the Delphi Method was used with the participation of 67 specialists from all regions of the country. After carrying out a selection of indicators based on bibliographic studies, the specialists were consulted in two stages, using descriptive statistics instruments. At the end of the consultation, 30 indicators were defined, divided into 5 dimensions, being: 9 in the technical dimension, 3 in the economic dimension, 9 in the environmental dimension, 5 in the social dimension and 4 in the political dimension. Subsequently, the indicators were applied in two municipalities in the state of Amazonas, through form submission with municipal managers. After obtaining the data and applying the respective weights, a percentage of $82.4 \%$ of need/opportunity was obtained for the implementation of a consortium between the two municipalities. However, there is a need to confirm this result with primary data and a survey of relative costs.
\end{abstract}

Keywords: Indicator; Waste; Sustainability; Amazon.

Topic: Planejamento, Gestão e Políticas Públicas Ambientais

Reviewed anonymously in the process of blind peer.
Received: 06/07/2021

Approved: $27 / 07 / 2021$
Rodrigo Couto Alves

Universidade Federal do Amazonas, Brasil http://lattes.cnpq.br/5580327610051854 http://orcid.org/0000-0002-7452-9455 rcouto@ufam.edu.br

Neliton Marques da Silva (iD

Universidade Federal do Amazonas, Brasil http://lattes.cnpq.br/2951411669963459

http://orcid.org/0000-0002-6812-729X

nmerinato@gmail.com

Evely Laranjeira Marques (iD)

Universidade Federal do Amazonas, Brasil http://lattes.cnpq.br/9575142539331285 http://orcid.org/0000-0001-7986-700X evelyl.marques@gmail.com

\section{Approved: $27 / 07 / 2021$}




\section{INTRODUÇÃO}

Os resíduos sólidos urbanos (RSU) são atualmente um dos principais problemas ambientais a serem equacionados, tendo atingido níveis críticos e emergenciais como por exemplo, no caso da ilha de lixo do Pacífico e da restrição imposta pela China quanto à importação de resíduos de países desenvolvidos.

No Brasil, o advento da Política Nacional de Resíduos Sólidos (PNRS), estabelecida pela Lei no 12.305/2010 e regulamentada pelo Decreto no 7.404/2010, marcou um grande avanço das políticas públicas nacionais em prol do desenvolvimento sustentável, ao estabelecer as diretrizes e os instrumentos para a gestão dos resíduos sólidos no país, além de apresentar vários instrumentos e metas para serem alcançados até 2014 (BRASIL, 2010).

Buscando promover o adequado gerenciamento dos resíduos sólidos urbanos nos municípios, a PNRS, em seu artigo 19, inciso III, exige a identificação das possibilidades de implantação de soluções consorciadas entre municípios considerando os critérios de economia de escala, a proximidade entre os mesmos e as formas de prevenção dos riscos ambientais.

O estado do Amazonas, ainda que disponha da Política Estadual de Resíduos Sólidos do Amazonas (PERS/AM), estabelecida pela Lei no 4.457/2017, se apresenta como um dos grandes geradores de RSU da região norte do país e, de seus 62 municípios, apenas Manaus não dispõe seus RSU em lixões. Esse quadro é acompanhado pela maioria dos municípios da Amazônia brasileira (SNIS, 2018), que apresenta dificuldades em equalizar esse problema.

Os Consórcios Públicos Intermunicipais para a Gestão de Resíduos Sólidos (CPIGRS), segundo Ferreira et al. (2017), surgem como alternativa administrativa e operacional, especialmente para os municípios de pequeno porte, pela dificuldade que enfrentam para eliminar seus lixões e construir aterros sanitários, principalmente por questões financeiras. Os consórcios permitem a diminuição dos custos associados ao atendimento às normas ambientais, assim como conseguem alcançar um ganho de escala por possibilitar o atendimento de um maior número de municípios.

Contudo, a análise de viabilidade de um CPIGRS para os municípios da Amazônia não pode simplesmente replicar os modelos adotados em outras regiões do país. Segundo Lima (2003), os modelos que são replicados sem considerar as especificidades da região onde será implantado, tendem a não se adaptarem e se tornam instáveis em sua amplificação, além de falharam em seu gerenciamento.

Mediante o exposto, cabe a questionar se seria técnica, econômica, social, política e ambientalmente viável a implantação de CPIGRS no Amazonas. O mérito do presente estudo encontra-se na definição de indicadores que possibilitarão a análise da viabilidade da implantação de consórcios públicos intermunicipais para a gestão dos resíduos sólidos no Amazonas.

\section{MATERIAIS E MÉTODOS}

Os municípios selecionados para o estudo foram Silves e Itapiranga, no estado do Amazonas, localizados na Região Metropolitana de Manaus e na Microrregião de Itacoatiara. A população de Silves e 
Itapiranga são, respectivamente, 9.171 e 9.148 habitantes. A metodologia adotada na definição dos indicadores utilizados para analisar a viabilidade da implantação de um CPIGRS nos municípios pode ser resumida conforme fluxograma (Figura 1).

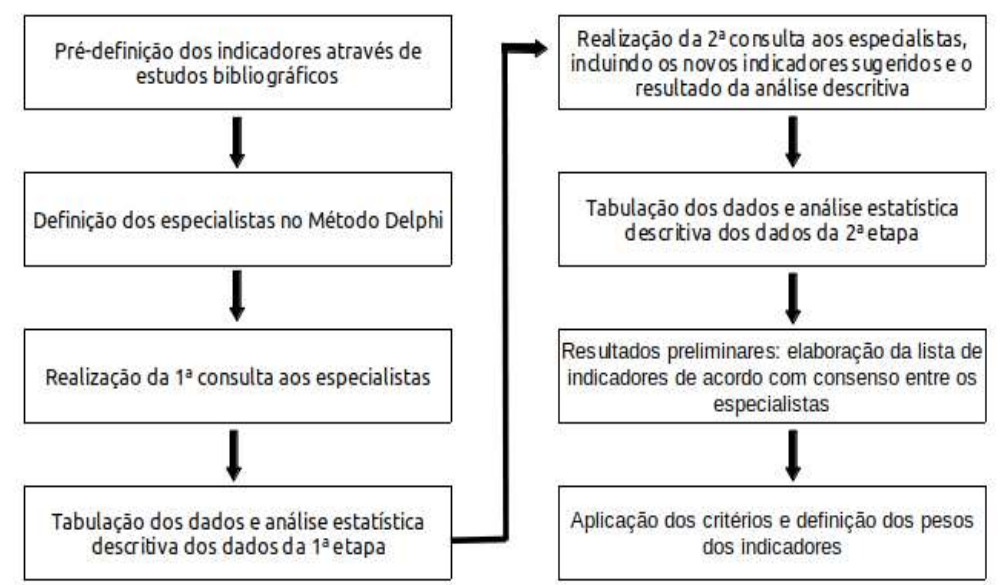

Figura 1: Fluxograma do Método Delphi aplicado no estudo.

Inicialmente, foi realizado um estudo bibliográfico para a seleção prévia de indicadores que abordassem a implantação, manutenção ou eficácia dos modelos de consórcios públicos intermunicipais que já estejam implantados no país. Dentre os métodos consolidados para a definição de indicadores, o escolhido para este estudo foi o Método Delphi.

Segundo Lindstone et al. (2002) esse método consiste na utilização da opinião de profissionais especialistas em um determinado assunto para obtenção de consenso sobre determinada situação, que deve ser obtido através de aplicação de rodadas de aplicação de questionários alternados para obtenção de respostas qualificadas.

O método consiste numa técnica de processo coletivo que objetiva obter, comparar e direcionar a opinião de especialistas para um consenso em um determinado assunto (SPÍNOLA, 1984). De forma concisa, essa técnica é executada por meio de questionários que são reencaminhados até uma certa convergência dos resultados. Este método foi aplicado com o auxílio da plataforma 'Google Formulários'. Para o recrutamento dos especialistas em resíduos sólidos, inicialmente foi enviado um e-mail individualizado contendo o objetivo do estudo e o link para a página do formulário. Na parte inicial do formulário foi inserido o Termo de Consentimento Livre e Esclarecido (TCLE).

Para a definição dos especialistas utilizou-se como fonte: programas de pós-graduação, anais dos eventos (congressos, simpósios) de resíduos sólidos dos últimos anos, periódicos com publicação na área, institutos, públicos ou privados, de limpeza pública e/ou saneamento básico, inclusive alguns consórcios. Para a aplicação do método, foi definida uma amostra de 129 especialistas de todas as regiões do país.

Os especialistas foram categorizados em atuação e área de conhecimento. Quanto à atuação, os mesmos foram classificados como acadêmicos, pesquisadores e profissionais do setor. Foram considerados acadêmicos os especialistas que atuam em universidades, públicas ou privadas, e que estão na ativa. Os pesquisadores são os acadêmicos que não estão mais na ativa ou que atuam em órgãos de pesquisa. E os profissionais são os consultores, servidores públicos ou empregados da iniciativa privada que atuam fora do 
ambiente acadêmico e de pesquisa.

Os especialistas foram divididos por áreas de conhecimento, sendo elas divididas em três: resíduos sólidos, saneamento básico e meio ambiente. Os especialistas que tivessem maior parte de sua obra relacionada especificamente com resíduos foram enquadrados na primeira categoria. Aqueles especialistas que tinham seus trabalhos divididos nas áreas do saneamento (resíduos sólidos, água para abastecimento, águas residuárias e drenagem urbana) foram categorizados na segunda classificação. Os especialistas que trabalham em diversas áreas que envolvem o meio ambiente (impactos ambientais, serviços ambientais, educação ambiental) incluindo resíduos sólidos, foram categorizados na terceira categoria.

Após pré-selecionados, os indicadores foram inseridos na plataforma supracitada, sendo realizados alguns testes para verificar sua funcionalidade e recursos necessários para a análise. Posteriormente, iniciouse a aplicação da primeira consulta no Método Delphi. Ressalta-se que para melhor eficácia do método é necessário o anonimato dos especialistas para que não ocorra influência de especialistas renomados na área.

Para a classificação dos indicadores foi utilizada a Escala de Likert, em que o especialista escolhe uma das alternativas objetivando aumentar a amplitude de expressão entre os pares, para se avaliar o nível de concordância ou discordância entre eles, definindo-se assim o grau de aceitação de cada indicador (VEIGA et al., 2013). A Escala foi assim definida em cinco níveis, a saber: 1) Muito Importante; 2) Importante; 3) Desejável; 4) Não Prioritário; 5) Dispensável.

Na primeira consulta, os formulários foram enviados, admitindo-se 20 dias como prazo limite para recebimento das respostas. Após o recebimento das respostas, foram extraídas a mediana, moda e frequência relativa de cada indicador, sendo também realizada a seleção dos novos indicadores sugeridos pelos especialistas. Na segunda rodada, foi encaminhado novo formulário, incluindo os novos indicadores sugeridos, adicionando-se o resultado da análise estatística descritiva da primeira consulta. Admitiram-se 20 dias como prazo limite.

Após o recebimento das respostas, novamente foi realizada a análise estatística descritiva. Foram eliminados os indicadores que tiveram as modas inferior a 4 e que apresentaram frequência relativa inferior a $75 \%$ (dentre os indicadores cuja moda foi igual ou superior a 4), assim como os indicadores que tinham similaridade ou de mesma abrangência. O peso foi definido utilizando-se parcialmente a metodologia de Ribeiro (2005), ou seja, através do percentual da frequência relativa, sendo que estas foram ponderadas em função da quantidade de indicadores de uma mesma dimensão. Posteriormente, criou-se um questionário com os indicadores que foi aplicado em entrevistas com gestores públicos dos municípios de Silves e Itapiranga em outubro de 2019.

\section{RESULTADOS E DISCUSSÃO}

Após a análise bibliográfica da literatura científica sobre os consórcios, verificou-se que na implantação de um CPIGRS é necessário se ter uma visão estratégica sobre os aspectos econômicos, ambientais, sociais e políticos (FERREIRA et al., 2017) e que o sistema funcione de forma transparente (SILVEIRA, 2008). Segundo a Política Nacional de Resíduos Sólidos, a gestão integrada conceitua-se como: 
"conjunto de ações voltadas para a busca de soluções para os resíduos sólidos, de forma a considerar as dimensões política, econômica, ambiental, cultural e social, com controle social e sob a premissa do desenvolvimento sustentável" (BRASIL, 2010).

A questão econômica é um dos aspectos que pode ser beneficiada com a implantação de um CPIGRS. A PNRS, em seu artigo 45, o Decreto no 7.404/2010, em seu artigo 79 e o PERS-AM, em seu artigo 13, preveem a prioridade na obtenção dos incentivos instituídos pelo Governo Federal e Estadual, respectivamente, para os municípios que estiverem participando de um CPIGRS. Assim como a PNRS prevê também, em seu artigo $8^{\circ}$, o ganho de escala, o que foi ratificado pelos estudos sobre consorciamento entre entes federados (BARROS, 2017; CALDERAN, 2013; FURTADO, 2017; MORAES, 2012).

Ambientalmente, os estudos apontaram que a implantação de um consórcio público beneficia o meio ambiente principalmente pela diminuição dos rejeitos (NOVAKOWSKI et al., 2017), maior vida útil do aterro (SUKUZl et al., 2009), aumento da coleta seletiva e reciclagem (CALDERAN, 2013) e maior conscientização ambiental da população (FONSECA, 2010).

Socialmente, os autores apontam a importância da inclusão das associações e cooperativas de catadores (NOVAKOWSKI et al., 2017; FERREIRA et al., 2017). Silveira (2008) destaca a necessidade de o CPIGRS não focar apenas no manejo, mas também a relação entre as partes que participam desse processo de gestão.

Segundo Fonseca (2010) é no campo político que são definidas as características e perspectivas da sustentabilidade urbana, pois são os atores políticos que determinam as ações do Estado e, consequentemente, dos que habitam a cidade, através de normas legais e políticas públicas. Contudo, se houver convergência de esforços entre os prefeitos devido a divergências político-partidários, a gestão compartilhada dos resíduos sólidos não será eficaz, independente da viabilidade técnica, econômica e ambiental da gestão dos RSU (MACEDO JÚNIOR, 2014). Segundo Oliveira (2004), são necessárias conversas entre todos os entes políticos para equacionar o problema da divergência partidária.

Alguns estudos ratificaram a necessidade de uma análise sobre as dimensões técnica, econômica, social, ambiental e política (SILVEIRA, 2008; FERREIRA et al., 2008; SUZUKI, 2009; FONSECA, 2010; ABREU et al., 2016). Partindo desse pressuposto, foram estabelecidas listas de indicadores, a partir dos estudos supracitados, que contemplassem essas cinco dimensões (Quadro 2 ao 6). Inicialmente, os indicadores irão possuir variáveis binárias, uma positiva e outra negativa. Sendo assim, quanto maior a quantidade de respostas negativas, maior a necessidade (ou oportunidade) de implementar um CPIGRS.

Quadro 1: Indicadores pré-selecionados na dimensão técnica para implementação de CPIGRS.

\begin{tabular}{ll}
\hline Código & Dimensão Técnica \\
\hline TC-1 & A proximidade física das sedes municipais é maior que 50km via malha rodoviária. \\
TC-2 & Inexistência de conexão entre as sedes via malha rodoviária. \\
TC-3 & Existência de recursos humanos municipais qualificados para a gestão dos RSU. \\
TC-4 & Existência de regulação e fiscalização dos serviços de gestão dos RSU. \\
TC-5 & Existência de embarcações para um eventual transporte fluvial de RSU. \\
TC-6 & Existência de capacitação técnica específica sobre RSU para os funcionários do setor. \\
TC-7 & Universalização da coleta de RSU. \\
TC-8 & Existência de galpão de triagem no município. \\
TC-9 & Existência de procedimentos operacionais e especificações mínimas para o sistema de coleta de RSU.
\end{tabular}


TC-10 Existência de recursos técnicos municipais (profissionais, maquinaria, frota...).

TC-11 Disponibilidade de área (terreno próprio) para disposição final de acordo com as diretrizes legais.

Quadro 2: Indicadores pré-selecionados na dimensão econômica para implementação de CPIGRS.

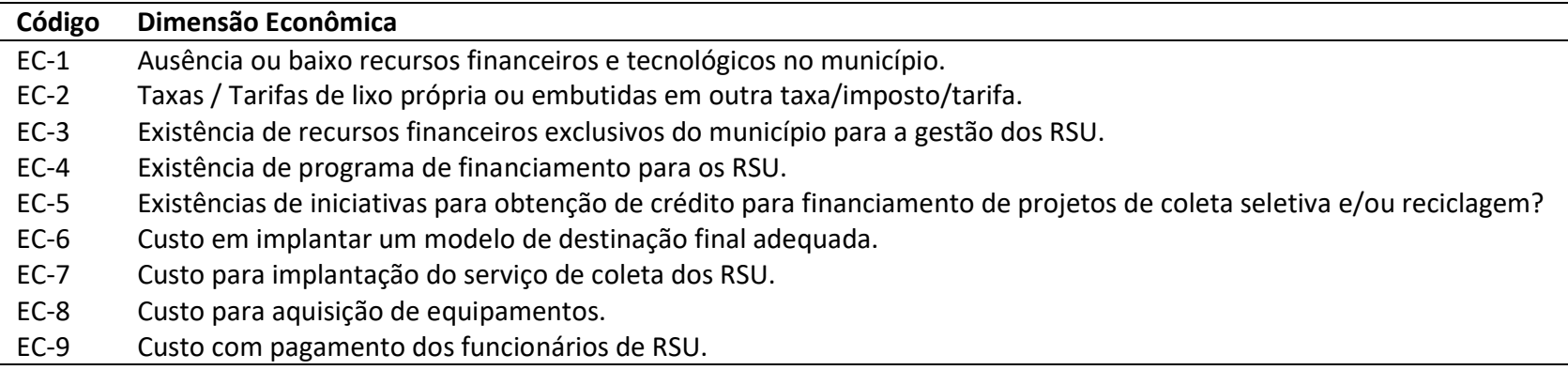

Quadro 3: Indicadores pré-selecionados na dimensão ambiental para implementação de CPIGRS.

\begin{tabular}{ll}
\hline Código & Dimensão Ambiental \\
\hline AM-1 & Grau de ocorrência de despejo de resíduos em lixões. \\
AM-2 & Nível da capacidade de recebimento de RSU do local de destinação final. \\
AM-3 & Grau de recuperação de materiais recicláveis através da coleta seletiva. \\
AM-4 & Existência de metas de coleta seletiva e reciclagem dos resíduos. \\
AM-5 & Existência de plano para recuperação da área degradada (lixão). \\
AM-6 & Existência de Termo de Ajustamento de Conduta Ambiental - TACA referente ao lixão. \\
AM-7 & Existência de programa de educação ambiental continuada. \\
AM-8 & Existência de convênios de logística reversa para algum tipo de RSU. \\
AM-9 & Ausência de descarte irregular de RSU (lixeiras viciadas). \\
AM-10 & Existência de manejo adequado dos RSS e RCC. \\
\hline
\end{tabular}

Quadro 4: Indicadores pré-selecionados na dimensão social para implementação de CPIGRS.

\begin{tabular}{ll}
\hline Código & Dimensão Social \\
\hline SC-1 & Existência de catadores vivendo nos locais de disposição de RSU. \\
SC-2 & Existência de cooperativa/associação de catadores no município. \\
SC-3 & $\begin{array}{l}\text { Existência de programas de apoio e incentivo para a formação e desenvolvimento de cooperativas/associação de } \\
\text { catadores. }\end{array}$ \\
SC-4 & $\begin{array}{l}\text { Existência de programas de inclusão social e remuneração para catadores cooperados. } \\
\text { SC-5 }\end{array}$ \\
SC-6 & Presença de entidades de ensino técnico, graduação e pós-graduação nos municípios. \\
\hline
\end{tabular}

Quadro 5: Indicadores pré-selecionados na dimensão política para implementação de CPIGRS.

\begin{tabular}{ll}
\hline Código & Dimensão Política \\
\hline PL-1 & Inexistência de interesses comuns entre os municípios quanto aos RSU. \\
PL-2 & Indisposição de cooperação por parte dos prefeitos quanto aos RSU. \\
PL-3 & Inexistência da busca pela superação de conflitos políticos partidários. \\
PL-4 & Tomada de decisão política em se consorciar. \\
PL-5 & Existência de uma identidade intermunicipal. \\
PL-6 & Existência de conflito de interesses político-partidários. \\
PL-7 & Os municípios fazem parte do mesmo Arranjo Territorial Ótimo (ATO). \\
PL-8 & Os municípios fazem parte da mesma bacia hidrográfica? \\
PL-9 & Inexistência do consórcio público como solução compartilhada no PMGIRS \\
\hline
\end{tabular}

Dos 129 especialistas consultados, 68 responderam o questionário (Apêndice II), totalizando um retorno de 52,7\%. Em comparação a outros trabalhos que utilizaram essa técnica, o percentual do retorno foi satisfatório, pois Coelho et al. (2011) teve um retorno de 55,7\%, Vimieiro (2009) de 44,2\%, Souza e Libânio (2009) de 75\%, Padilha (2005) de 56\% e Ribeiro (2005) de 60\%. Essa participação foi considerada satisfatória e ocorreu pela qualidade do questionário no que diz respeito a facilidade e rapidez no seu preenchimento.

Em relação às respostas, o maior percentual correspondeu a Região Norte (29\%), seguido das regiões Sul (25\%), Sudeste (22\%) e Nordeste (21\%), conforme Tabela 2. A região Centro-Oeste teve participação baixa, com apenas $3 \%$ dos especialistas. 
Tabela 1: Distribuição percentual das respostas dos especialistas por região geográfica no Brasil.

\begin{tabular}{|c|c|c|c|c|c|c|c|c|c|c|}
\hline \multirow{3}{*}{ Região Brasileira } & \multicolumn{10}{|c|}{ Especialistas } \\
\hline & \multicolumn{3}{|c|}{ Acadêmico } & \multicolumn{3}{|c|}{ Pesquisador } & \multicolumn{3}{|c|}{ Profissional } & \multirow[t]{2}{*}{ Total de Especialistas } \\
\hline & RS & SB & MA & RS & SB & MA & RS & SB & MA & \\
\hline Norte & $3 \%$ & $5 \%$ & $12 \%$ & $3 \%$ & $0 \%$ & $1,5 \%$ & $1,5 \%$ & $0 \%$ & $3 \%$ & $29 \%$ \\
\hline Sul & $3 \%$ & $5 \%$ & $6 \%$ & $0 \%$ & $1 \%$ & $0 \%$ & $7 \%$ & $0 \%$ & $3 \%$ & $25 \%$ \\
\hline Sudeste & $6 \%$ & $1 \%$ & $6 \%$ & $0 \%$ & $0 \%$ & $4,5 \%$ & $3 \%$ & $1,5 \%$ & $0 \%$ & $22 \%$ \\
\hline Nordeste & $5 \%$ & $9 \%$ & $3 \%$ & $0 \%$ & $0 \%$ & $0 \%$ & $4 \%$ & $0 \%$ & $0 \%$ & $21 \%$ \\
\hline Centro-oeste & $0 \%$ & $3 \%$ & $0 \%$ & $0 \%$ & $0 \%$ & $0 \%$ & $0 \%$ & $0 \%$ & $0 \%$ & $3 \%$ \\
\hline Total & $17 \%$ & $23 \%$ & $27 \%$ & $3 \%$ & $1 \%$ & $6 \%$ & $15,5 \%$ & $1,5 \%$ & $6 \%$ & $100 \%$ \\
\hline
\end{tabular}

RS: Resíduos Sólidos; SB: Saneamento Básico; MA: Meio Ambiente.

Quanto às categorias, os acadêmicos tiveram maior participação, com $67,2 \%$, seguidos dos profissionais da área que corresponderam por $22,4 \%$ e a menor participação foram as dos classificados como pesquisadores, com apenas 10,5\%. Quanto às áreas, houve um certo equilíbrio, sendo a área de meio ambiente a que teve maior participação com $38,9 \%$, seguida da área de resíduos sólidos com $34,3 \%$ e por fim, a área de saneamento que correspondeu por $26,9 \%$.

Após recebimento das respostas da 1a etapa, foram acatadas algumas novas sugestões de indicadores, conforme Quadros 7 ao 10. Não houve sugestões de indicadores para a dimensão política.

Quadro 6: Indicadores sugeridos pelos especialistas para a dimensão técnica.

\begin{tabular}{ll}
\hline Código & Dimensão Técnica \\
\hline TC-12 & Grau de cobertura de coleta seletiva. \\
TC-13 & Grau de deformações na topografia do translado entre as sedes municipais e o aterro. \\
TC-14 & Grau de recuperação de materiais recicláveis. \\
TC-15 & Existência de conexão entre as sedes via Fluvial. \\
TC-16 & Existência de Unidade de Transbordo. \\
TC-17 & Variação da geração per capita de RSU. \\
\hline
\end{tabular}

Quadro 7: Indicadores sugeridos pelos especialistas para a dimensão econômica.

\begin{tabular}{ll}
\hline Código & Dimensão Econômica \\
\hline EC-10 & Existência de mercado para compras de materiais recicláveis. \\
\hline EC-11 & Percentual do pagamento de impostos públicos que são destinados a ações de GRS. \\
\hline EC-12 & Existência de estudos locais sobre alternativas para a destinação dos RSU. \\
\hline EC-13 & Custo atual do sistema de manejo (R\$/hab.ano) \\
\hline
\end{tabular}

Quadro 8: Indicadores sugeridos pelos especialistas para a dimensão ambiental.

\begin{tabular}{ll}
\hline Código & Dimensão Ambiental \\
\hline AM-11 & Existência de lixões em área urbana ou próximo de aeroportos. \\
\hline AM-12 & Existência de cursos de capacitação de profissionais de RSU. \\
\hline AM-13 & Existência de lei de grande gerador. \\
\hline AM-14 & Existência de aterro sanitário no município, em conformidade com as normas técnicas e legais. \\
\hline AM-15 & Grau de recuperação de passivos ambientais (antigos lixões). \\
\hline
\end{tabular}

Quadro 9: Indicadores sugeridos pelos especialistas para a dimensão social.

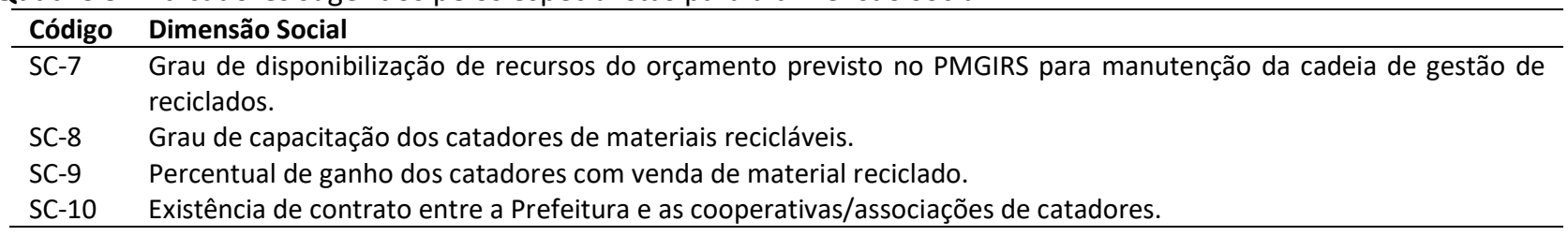

Foram encaminhados para a 2a etapa do método um total de 64 indicadores, sendo: 17 na dimensão técnica, 13 na dimensão econômica, 15 na dimensão ambiental, 10 na dimensão social e 9 na dimensão política. O primeiro critério aplicado para a definição dos indicadores foi a moda. Aqueles que tiveram a moda abaixo de 4 foram excluídos, o que eliminou apenas 5 indicadores. 
O segundo critério aplicado foi considerar apenas os indicadores cuja frequência relativa das modas 4 e 5 situassem acima do 3ำ quartil, ou seja, que tivessem a preferência de $75 \%$ dos especialistas. Após esse critério foram excluídos mais 21 indicadores.

O terceiro critério aplicado foi a análise da similaridade/abrangência dos indicadores entre si, sujeitando um ao escopo de outro. Nesta etapa foram excluídos 8 indicadores, conforme abaixo: - TC-5: rejeitado pois permite sua abrangência do proposto no indicador TC-10; - EC-1: rejeitado pois permite sua abrangência do proposto no indicador EC-3; - EC-6/EC-7/EC-9: rejeitados pois permitem sua abrangência do proposto no indicador EC-13; - AM-1: rejeitado pois permite sua abrangência do proposto no indicador AM14; - AM-2: rejeitado pois sugere que o município possui um aterro sanitário e não um lixão; - PL-4: rejeitado pois permite sua abrangência do proposto no indicador PL-2 além de possuir difícil mensuração.

Ao término dos critérios, restaram 30 indicadores, conforme apresenta o Gráfico 1, sendo: - 9 na dimensão técnica: TC-1, TC-2, TC-3, TC-4, TC-6, TC-7, TC-9, TC-10 e TC-11; - 3 na dimensão econômica: EC-3, EC-5 e EC13; - 9 na dimensão ambiental: AM-3, AM-4, AM-5, AM-6, AM-7, AM-8, AM-9, AM-10 e AM-14; - 5 na dimensão social: SC-1, SC-2, SC-3, SC-4 e SC-9; - 4 na dimensão política: PL-1, PL-2, PL-3 e PL-9.

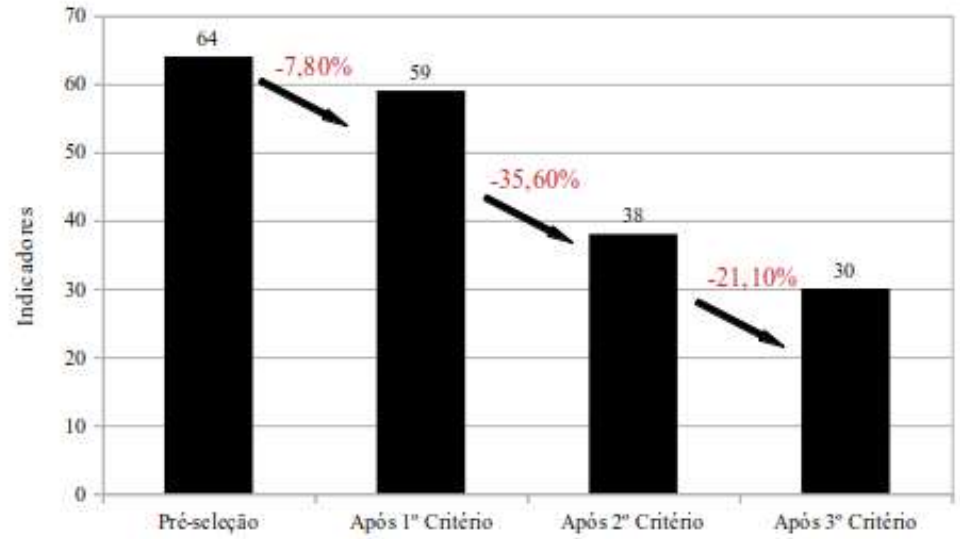

Gráfico 1: Quantidade de indicadores selecionados após aplicação dos critérios.

Observou-se que, como 70\% dos especialistas são de regiões do país que não a amazônica, talvez por essa razão, alguns indicadores técnicos que tratam de características amazônicas não foram considerados, a exemplo dos indicadores TC-5 e TC15, que estão relacionados ao modal fluvial. Ressalta-se que mais de $90 \%$ dos municípios amazonenses encontram-se na calha dos rios e que o principal acesso aos meios é via fluvial, assim como poucos municípios amazonenses possuem ligação via modal rodoviário.

O indicador TC-1, quase foi desconsiderado (obteve o valor mínimo necessário do segundo critério), contudo é de considerável relevância no estado do Amazonas devido às grandes distâncias entre as sedes municipais, diferente do que ocorre em outras regiões do país.

Para a importância relativa de cada indicador, os pesos foram determinados a partir do percentual da frequência relativa destes indicadores dentro de sua respectiva dimensão, sendo o resultado ajustado para a base 1000 (Tabela 2). 
Tabela 2: Definição do peso dos indicadores.

\begin{tabular}{|c|c|c|c|c|c|}
\hline Dimensão & Indicador & Frequência Relativa (\%) & Coeficiente ponderado & Coeficiente na base 1000 & Peso do indicador (\%) \\
\hline \multirow{9}{*}{ TÉCNICA } & TC-1 & 0,75 & 0,083 & 0,02 & 1,97 \\
\hline & TC-2 & 0,86 & 0,095 & 0,023 & 2,26 \\
\hline & TC-3 & 0,87 & 0,097 & 0,023 & 2,3 \\
\hline & TC-4 & 0,87 & 0,097 & 0,023 & 2,3 \\
\hline & TC-6 & 0,86 & 0,095 & 0,023 & 2,26 \\
\hline & TC-7 & 0,92 & 0,102 & 0,024 & 2,43 \\
\hline & TC-9 & 0,87 & 0,097 & 0,023 & 2,3 \\
\hline & TC-10 & 0,84 & 0,093 & 0,022 & 2,22 \\
\hline & TC-11 & 0,78 & 0,086 & 0,02 & 2,05 \\
\hline \multirow{3}{*}{ ECONÔMICA } & $\mathrm{EC}-3$ & 0,79 & 0,265 & 0,063 & 6,27 \\
\hline & EC-5 & 0,75 & 0,249 & 0,059 & 5,9 \\
\hline & EC-13 & 0,94 & 0,312 & 0,074 & 7,4 \\
\hline \multirow{9}{*}{ AMBIENTAL } & AM-3 & 0,87 & 0,097 & 0,023 & 2,3 \\
\hline & AM-4 & 0,89 & 0,099 & 0,023 & 2,34 \\
\hline & AM-5 & 0,86 & 0,095 & 0,023 & 2,26 \\
\hline & AM-6 & 0,81 & 0,09 & 0,021 & 2,13 \\
\hline & AM-7 & 0,78 & 0,086 & 0,02 & 2,05 \\
\hline & AM-8 & 0,78 & 0,086 & 0,02 & 2,05 \\
\hline & AM-9 & 0,83 & 0,092 & 0,022 & 2,17 \\
\hline & AM-10 & 0,87 & 0,097 & 0,023 & 2,3 \\
\hline & AM-14 & 1 & 0,111 & 0,026 & 2,63 \\
\hline \multirow{5}{*}{ SOCIAL } & SC-1 & 0,9 & 0,181 & 0,043 & 4,29 \\
\hline & SC-2 & 0,79 & 0,159 & 0,038 & 3,76 \\
\hline & SC-3 & 0,78 & 0,156 & 0,037 & 3,69 \\
\hline & SC-4 & 0,78 & 0,156 & 0,037 & 3,69 \\
\hline & SC-9 & 0,94 & 0,187 & 0,044 & 4,44 \\
\hline \multirow{4}{*}{ POLÍTICA } & PL-1 & 0,9 & 0,226 & 0,054 & 5,36 \\
\hline & PL-2 & 0,83 & 0,206 & 0,049 & 4,89 \\
\hline & PL-3 & 0,84 & 0,21 & 0,05 & 4,99 \\
\hline & PL-9 & 0,84 & 0,21 & 0,05 & 4,99 \\
\hline
\end{tabular}

Assim, a importância relativa de cada dimensão para a análise da viabilidade de uma CPIGRS será: técnica $(20,08 \%)$, econômica $(19,57 \%)$, ambiental $(20,24 \%)$, social $(19,87 \%)$ e política $(20,23 \%)$. Neste primeiro momento, as variáveis dos indicadores possuirão apenas duas medidas contrárias. A pontuação foi calculada em cima do produto entre a média de cada município, considerando a pontuação de 1 para uma resposta negativa (N) e 0 para uma resposta positiva (S), com o respectivo peso do indicador (Tabela 3).

Tabela 3: Matriz de viabilidade de implementação de um CPIGRS entre Silves-AM e Itapiranga-AM.

\begin{tabular}{lllll}
\hline Ind. & Variáveis & Silves & Itapiranga & Ponto \\
\hline TC-1 & $\begin{array}{l}\text { (S) É maior que } 50 \mathrm{~km} \\
\text { (N) É menor ou igual a } 50 \mathrm{~km}\end{array}$ & $\mathrm{~N}$ & $\mathrm{~N}$ & 0,020 \\
\hline TC-2 & $\begin{array}{l}\text { (S) É inexistente a conexão rodoviária } \\
\text { (N) Existe conexão rodoviária }\end{array}$ & $\mathrm{N}$ & $\mathrm{N}$ & 0,023 \\
\hline TC-3 & $\begin{array}{l}\text { (S) Existência de um profissional qualificado no quadro de servidores } \\
\text { (N) Ausência de qualquer profissional qualificado no quadro de servidores }\end{array}$ & $\mathrm{N}$ & $\mathrm{N}$ & \multirow{2}{*}{0,023} \\
\hline
\end{tabular}


Ind. Variáveis

Silves Itapiranga Ponto

(S) Existência de relatórios e diagnósticos provenientes da fiscalização sobre a gestão

dos RSU

TC-4 (N) Inexistência de relatórios e diagnósticos provenientes da fiscalização sobre a gestão

$\mathrm{N} \quad \mathrm{N}$

0,023 dos RSU

(S) Ministração de cursos, internos ou externos, para os funcionários responsáveis pelo manejo de RSU

TC-6 (N) Não há ministração de cursos, internos ou externos, para os funcionários

$\mathrm{N} \quad \mathrm{N}$ responsáveis pelo manejo de RSU

\begin{tabular}{llll}
\hline TC-7 & $\begin{array}{l}\text { (S) O município possui mais de 90\% de universalização de coleta } \\
\text { (N) O município possui menos de 90\% de universalização de coleta }\end{array}$ & $\mathrm{S}$ & $\mathrm{S}$ \\
\hline TC-9 & $\begin{array}{l}\text { (S) Existência de um PMGIRS aprovado } \\
\text { (N) Ausência de um PMGIRS aprovado }\end{array}$ & $\mathrm{N}$ & $\mathrm{N}$ \\
\hline
\end{tabular}

(S) Existência de uma estrutura básica (funcionários específicos, tratores, caminhões,

TC- embarcações etc.)

10 (N) Ausência de uma estrutura básica (funcionários específicos, tratores, caminhões, embarcações etc.)

(S) Existência de área suficiente para a disposição de RSU dentro do município que

TC- atenda as diretrizes legais

11 (N) Ausência de área suficiente para a disposição de RSU dentro do município que atenda as diretrizes legais

EC-3 (S) O município possui receita dentro do orçamento anual para a gestão do RSU

(N) O município não possui receita dentro do orçamento anual para a gestão do RSU $\quad \begin{array}{llll}\text { N } & N & 0,063\end{array}$

(S) O município participa de editais ou outros meios para aquisição de crédito a órgãos

$\begin{array}{ll}\text { EC-5 } & \text { públicos ou privados } \\ & \text { (N) O município não participa de editais ou outros meios para aquisição de crédito a }\end{array}$ órgãos públicos ou privados

\begin{tabular}{|c|c|c|c|c|}
\hline $\begin{array}{l}\text { EC- } \\
13\end{array}$ & $\begin{array}{l}\text { (S) O custo é inferior ao disponível no orçamento do município } \\
\text { (N) O custo é igual ou superior ao disponível no orçamento do município }\end{array}$ & $\mathrm{N}$ & $\mathrm{N}$ & 0,074 \\
\hline AM-3 & $\begin{array}{l}\text { (S) Recuperação per capita de recicláveis secos igual ou maior que 4,7kg.hab/ano* } \\
\text { (N) Recuperação per capita de recicláveis secos menos que 4,7 kg.hab./ano* }\end{array}$ & $\mathrm{N}$ & $\mathrm{N}$ & 0,023 \\
\hline AM-4 & $\begin{array}{l}\text { (S) Existência de metas estabelecidas no PMGIRS } \\
\text { (N) Inexistência de metas estabelecidas no PMGIRS }\end{array}$ & $\mathrm{N}$ & $\mathrm{N}$ & 0,023 \\
\hline
\end{tabular}

(S) Existência de um plano de recuperação de área degradada do sistema de disposição

AM-5 de resíduos do município
(N) Inexistência de um plano de recuperação de área degradada do sistema de disposição de resíduos do município

(S) Existência de um, ou mais, TACA municipais referente ao sistema de disposição de

$\begin{array}{ll}\text { AM-6 resíduos } & \text { (N) Inexistência de TACA municipais referente ao sistema de disposição de resíduos }\end{array}$

\begin{tabular}{|c|c|c|c|c|}
\hline AM-7 & $\begin{array}{l}\text { (S) Existência de um programa de educação ambiental implantada no município } \\
\text { (N) Inexistência de um programa de educação ambiental implantada no município }\end{array}$ & $S$ & $S$ & 0,000 \\
\hline AM-8 & $\begin{array}{l}\text { (S) Existência de convênios de logística reversa para algum tipo de resíduo } \\
\text { (N) Inexistência de convênios de logística reversa para algum tipo de resíduo }\end{array}$ & $\mathrm{N}$ & $\mathrm{N}$ & 0,020 \\
\hline AM-9 & $\begin{array}{l}\text { (S) Ausência de lixeiras viciadas espalhadas pelo município } \\
\text { (N) Existência de lixeiras viciadas espalhadas pelo município }\end{array}$ & $\mathrm{N}$ & $\mathrm{N}$ & 0,022 \\
\hline $\begin{array}{l}\text { AM- } \\
10\end{array}$ & $\begin{array}{l}\text { (S) O manejo dos RSS e RCC é realizado adequadamente } \\
\text { (N) O manejo dos RSS e RCC é realizado inadequadamente }\end{array}$ & $\mathrm{N}$ & $\mathrm{N}$ & 0,023 \\
\hline $\begin{array}{l}\text { AM- } \\
14\end{array}$ & $\begin{array}{l}\text { (S) Os RSU são dispostos em aterro sanitário ou tratados de forma ambientalmente } \\
\text { adequada } \\
\text { (N) Os RSU são dispostos em aterro controlado ou lixão. }\end{array}$ & $\mathrm{N}$ & $\mathrm{N}$ & 0,026 \\
\hline SC-1 & $\begin{array}{l}\text { (S) Os catadores residem fora do lixão e trabalham dentro de cooperativa/associação de } \\
\text { catadores } \\
\text { (N) Presença de catadores trabalhando e vivendo dentro dos lixões }\end{array}$ & $S$ & $\mathrm{~N}$ & 0,021 \\
\hline SC-2 & $\begin{array}{l}\text { (S) Existência de cooperativa/associação de catadores no município } \\
\text { (N) Os catadores trabalham de forma autônoma }\end{array}$ & $\mathrm{N}$ & $\mathrm{N}$ & 0,038 \\
\hline SC-3 & $\begin{array}{l}\text { (S) Ministração de cursos, internos ou externos, para as cooperativas/associações de } \\
\text { catadores fornecidos pelo município } \\
\text { (N) Não há nenhum programa de apoio e incentivo para a formação e desenvolvimento } \\
\text { das cooperativas/associações de catadores }\end{array}$ & $\mathrm{N}$ & $\mathrm{N}$ & 0,037 \\
\hline SC-4 & $\begin{array}{l}\text { (S) Existência de convênios entre a prefeitura e os catadores para o manejo dos RSU } \\
\text { (N) Inexistência de convênios entre a prefeitura e os catadores para o manejo dos RSU }\end{array}$ & $S$ & $\mathrm{~N}$ & 0,018 \\
\hline SC-9 & $\begin{array}{l}\text { (S) Existência de lucro na comercialização dos materiais reciclados } \\
\text { (N) Existência de prejuízo na comercialização dos materiais reciclados }\end{array}$ & $\mathrm{N}$ & $\mathrm{N}$ & 0,044 \\
\hline PL-1 & $\begin{array}{l}\text { (S) É inexistente a preocupação do gestor municipal apresentou preocupação quanto ao } \\
\text { correto manejo dos RSU }\end{array}$ & $\mathrm{N}$ & $\mathrm{N}$ & 0,054 \\
\hline
\end{tabular}




\begin{tabular}{|c|c|c|c|c|}
\hline Ind. & Variáveis & Silves & Itapiranga & Ponto \\
\hline & (N) O gestor municipal não apresentou preocupação quanto ao correto manejo dos RSU & & & \\
\hline PL-2 & $\begin{array}{l}\text { (S) O gestor municipal se indispôs em criar um consórcio } \\
\text { (N) O gestor municipal não se indispôs em criar um consórcio }\end{array}$ & $\mathrm{N}$ & $\mathrm{N}$ & 0,049 \\
\hline PL-3 & $\begin{array}{l}\text { (S) É inexistente a busca pela superação de conflitos políticos partidários } \\
\text { (N) O gestor busca superar qualquer conflito político partidário }\end{array}$ & $\mathrm{N}$ & $\mathrm{N}$ & 0,050 \\
\hline PL-9 & $\begin{array}{l}\text { (S) É inexistente a inclusão dos consórcios públicos como solução compartilhada no } \\
\text { PMGIRS } \\
\text { (N) Os consórcios públicos constam como solução compartilhada no PMGIRS }\end{array}$ & $\mathrm{N}$ & $\mathrm{N}$ & 0,050 \\
\hline
\end{tabular}

*média da Região Norte no Sistema Nacional de Informações sobre Saneamento (SNIS) de 2018.

Os consórcios públicos têm sido apontados como uma alternativa para a gestão integrada de resíduos sólidos entre os municípios de pequeno e médio porte. Estudos apontam que há ganhos econômicos nos municípios que implantam um consórcio intermunicipal para gerenciar e dispor seus resíduos sólidos em um aterro sanitário consorciado (SAIANI et al., 2010; LIMA, 2003).

A presença de consórcios públicos é mais visível na Região Sudeste, representando $57 \%$ dos consórcios existentes do país, enquanto a Região Norte representa apenas $5 \%$ dos consórcios implementados (SNIS, 2018). Em sua maioria, os consórcios são limitados principalmente pela distância entre os municípios, fator este que viabiliza ou não, a implementação dos projetos consorciados.

A análise de viabilidade de um CPIGRS para os municípios da Amazônia necessita de um modelo que atenda às suas especificidades e não apenas aplicar modelos existentes em outras regiões do país.

Após calculado, o valor final da matriz foi de 0,824 , ou seja, no total da soma dos indicadores, os municípios de Silves e Itapiranga alcançaram um percentual de $82,4 \%$, que significa que ambos têm considerável necessidade (e oportunidade) de implantação de um CPIGRS.

Os indicadores possuem, em sua maioria, critérios qualitativos, sugerindo certo grau de subjetividade nas respostas. Segundo Castro et al. (2015), a aplicação de critérios subjetivos atende à realidade do Estado, logo, é possível utilizá-los, principalmente, em municípios que carecem de dados quantitativos, realidade em que se encontram a maioria dos municípios do estado do Amazonas.

Contudo, como a maioria dos indicadores apresentam critérios subjetivos, faz-se necessário o levantamento de dados primários e secundários (custos e dados técnicos) para ratificar o resultado apresentado na matriz sobre a viabilidade da implantação de um CPIGRS entre estes municípios.

Os consórcios intermunicipais são "vistos como instrumentos de cooperação entre governos municipais localizados em uma determinada microrregião ou região" (LIMA, 2003). Para este autor, os consórcios caracterizam-se pela união entre os municípios para resolver problemas de interesse comum com a utilização dos recursos de cada esfera do poder.

\section{CONCLUSÕES}

O estado do Amazonas, quanto aos resíduos sólidos, apresenta uma realidade preocupante, visto que apenas a capital não dispõe seus resíduos em lixões. Considerando a dificuldade em criar e manter um sistema de gestão de resíduos de forma isolada, surge a necessidade de verificar a possibilidade da implantação de consórcios públicos intermunicipais, buscando reduzir os custos e eliminar a disposição 
inadequada dos resíduos.

O Método Delphi mostrou-se como uma ferramenta adequada na obtenção do consenso sobre uma determinada temática, tendo sido obtidas a participação de uma boa quantidade de especialistas e uma boa taxa de retorno. Foram selecionados 30 indicadores, em sua maioria qualitativos, que estão subdividos em cinco dimensões, com pesos similares entre si. Contudo, verificou-se que não foi dada a importância devida para as características da região amazônica por parte dos especialistas, sendo que alguns indicadores representativos foram eliminados do processo seletivo.

Os indicadores expressos na matriz de viabilidade resultaram em 82,4\%, indicando uma considerável necessidade/oportunidade para a implementação de um CPIGRS entre os municípios de Silves e Itapiranga. Entretanto, faz-se necessário validar essa matriz, com levantamento de dados primários e análise de custos relativos a gestão de resíduos nesses municípios.

\section{REFERÊNCIAS}

ABREU, B. R.; SIQUEIRA, G. W.; SOUZA, J. S.; SILVA, A. X.. Avaliação da Sustentabilidade de um Consórcio Intermunicipal de Resíduos Sólidos entre os Municípios de Benjamin Constant e Atalaia do Norte - AM. In: ABREU, B. R.; LEITE, J. C.; SOUZA, J. S.. Tópicos Especiais em Meio Ambiente: uma abordagem prática de temas sustentáveis. São Paulo: Embu das Artes: Alexa Cultural 2018. p.25-30.

AMAZONAS. Lei n. 4.457, de 12 de abril de 2017. Institui a Política Estadual de Resíduos Sólidos do Amazonas PERS/AM, e dá outras providências. Manaus: DOE, 2017.

BARROS, L. V. L.. Viabilidade da Implantação de Solução Consorciada para Gestão de Resíduos Sólidos na Região Metropolitana de Florianópolis (RMF). Monografia (Especialização em Gestão Ambiental) - Universidade Federal do Paraná, Curitiba, 2017.

BRASIL. Decreto n. 7.404, de 23 de dezembro de 2010. Regulamenta a Lei no 12.305, de 2 de agosto de 2010, que institui a Política Nacional de Resíduos Sólidos, cria o Comitê Interministerial da Política Nacional de Resíduos Sólidos e o Comitê Orientador para a Implantação dos Sistemas de Logística Reversa, e dá outras providências. Brasília: DOU, 2010.

BRASIL. Lei n. 12.305, de 2 de agosto de 2010. Institui a Política Nacional dos Resíduos Sólidos. Brasília: DOU, 2010.

BRASIL. Ministério do Desenvolvimento Regional. Secretaria Nacional de Saneamento Ambiental. Sistema Nacional de Informações sobre Saneamento: Diagnóstico do manejo de resíduos sólidos urbanos - 2018. Brasília: MCIDADES, 2018.

CALDERAN, T. B.. Consórcio Público Intermunicipal de Gerenciamento de Resíduos Sólidos Domésticos: Um estudo de caso. Dissertação (Mestrado em Ambiente e Desenvolvimento) - Centro Universitário Univates, Lajeado, 2013.

CASTRO, M. A. O.; SILVA, N. M.; MARCHAND, G. A. E. L.. Desenvolvendo indicadores para a gestão sustentável de resíduos sólidos nos municípios de Iranduba, Manacapuru e Novo Airão, Amazonas, Brasil. Eng. Sanit. Ambient., v.20, n.3, 2015.

FERREIRA, C. F. A.; JUCA, J. F. T.. Metodologia para avaliação dos consórcios de resíduos sólidos urbanos em Minas Gerais. Revista Engenharia Sanitária e Ambiental, Rio de Janeiro, v.22, n.3, p.513-521, 2017

FONSECA, D. R. M.. O Consórcio Público Intermunicipal como Instrumento de Gestão dos Resíduos Sólidos Urbanos: Um Estudo de Caso nos Municípios de Tucuruí e Breu Branco (Estado do Pará). Dissertação (Mestrado em Gestão dos Recursos Naturais e Desenvolvimento Local na Amazônia) - Universidade Federal do Pará, Belém, 2010.

FURTADO, E. D.. Consórcios Intermunicipais para Gestão de Resíduos Sólidos Urbanos: uma análise da implantação na Região Norte de Goiás - CIDENORTE. Dissertação (Mestrado em Geografia) - Universidade Federal do Tocantins, Porto Nacional, 2017.

LIMA, J. D.. Consórcio de Desenvolvimento Intermunicipal: Instrumento de Integração Regional. ABES, 2003

LINDSTONE, H. A.; TUROFF, M.. The Delphi Method: techniques and applications. 2002.

MACEDO JUNIOR, R. C.. Consórcio Intermunicipal para Gestão Ambiental nos Municípios de Pequeno Porte 1 e 2 do Nordeste Paraense: Uma possibilidade para Gestão Ambiental?. Dissertação (Mestrado em Gestão de Recursos Naturais e Desenvolvimento Local na Amazônia) Universidade Federal do Pará, Belém, 2014.

MORAES, J. L.. Os Consórcios Públicos e a Gestão Integrada de Resíduos Sólidos em Pequenos Municípios do Estado do Ceará, Brasil. Revista Geonorte, Edição Especial, Manaus, v.3, n.4, p.1171-1180, 2012.

NOVAKOWSKI, G. A. B.; TRINDADE, V.; PIOVEZANA, L.. Consórcios Intermunicipais para Gestão de Resíduos Sólidos: 
Estudo da Viabilidade Econômica no Município de Formosa do Sul (SC). In: SEMINÁRIO INTERNACIONAL SOBRE DESENVOLVIMENTO REGIONAL, 8. Anais. Santa Cruz do Sul, 2017.

OLIVEIRA, G.. Consórcio Intermunicipal para o Manejo Integrado de Lixo em Cinco Municípios da Região Administrativa de Bauru. Dissertação (Mestrado em Geografia) - Universidade Estadual Paulista, Rio Claro, 2004.

RIBEIRO, J.. Avaliação de Consórcios Públicos de Gestão de Resíduos Sólidos Urbanos: os Casos de João Monlevade e Ibirité (MG). Dissertação (Mestrado em Saneamento, Meio Ambiente e Recursos Hídricos) - Universidade Federal de Minas Gerais, Belo Horizonte, 2009.

SAIANI, C. C. S.; DOURADO, J.; JÚNIOR, R. T.. Resíduos Sólidos no Brasil: oportunidades e desafios da lei federal no 12.305 (lei de resíduos sólidos). Barueri: Minha, 2014.

SILVEIRA, R. C. E.. Gestão Consorciada de Resíduos Sólidos
Urbanos em Municípios de Pequeno Porte: uma contribuição para a sustentabilidade nas relações socioambientais. Tese (Doutorado em Engenharia Ambiental) - Universidade Federal de Santa Catarina, Florianópolis, 2008.

SNIS. Sistema Nacional de Informações sobre Saneamento. Diagnóstico do Manejo de Resíduos Sólidos Urbanos. SNIS 2018.

SPÍNOLA, A. W. P.. Delfos: proposta tecnológica alternativa. São Paulo: USP, 1984.

SUKUZI, J. A. N.; GOMES, J.. Consórcios intermunicipais para a destinação de RSU em Aterros Regionais: estudo prospective para os municípios no Estado do Paraná. Revista Engenharia Sanitária e Ambiental, v.14, n.2, p.155-158, 2009.

VEIGA, J. E.. Desenvolvimento sustentável, que bicho é esse?. Campinas: Autores associados, 2018.

A CBPC - Companhia Brasileira de Produção Científica (CNPJ: 11.221.422/0001-03) detém os direitos materiais desta publicação. Os direitos referem-se à publicação do trabalho em qualquer parte do mundo, incluindo os direitos às renovações, expansões e disseminações da contribuição, bem como outros direitos subsidiários. Todos os trabalhos publicados eletronicamente poderão posteriormente ser publicados em coletâneas impressas sob coordenação da Sustenere Publishing, da Companhia Brasileira de Produção Científica e seus parceiros autorizados. Os (as) autores (as) preservam os direitos autorais, mas não têm permissão para a publicação da contribuição em outro meio, impresso ou digital, em português ou em tradução. 